\title{
EXPLICIT FABER POLYNOMIALS ON CIRCULAR SECTORS
}

\author{
KARIN GATERMANN, CHRISTOPH HOFFMANN, AND GERHARD OPFER
}

\begin{abstract}
We present explicit and precise expressions for the Faber polynomials on circular sectors up to degree 20 . The precision is obtained by modifying (and simultaneously speeding up) an algorithm of Coleman and Smith so that an essential part of the Faber polynomials can be represented using only rational numbers. The growth of the coefficients of the Faber polynomials is determined. In addition, explicit expressions are given for the area two-norm and line two-norm of these polynomials. A conjecture is stated with respect to the uniform (infinity) norm which would also allow the explicit computation of the corresponding uniform norms of the Faber polynomials. Apart from a table of Faber polynomials, there are several other tables and graphs that illustrate the behavior of the Faber polynomials.
\end{abstract}

\section{INTRODUCTION}

Let $S \subset \mathbb{C}$ be a compact set whose complement admits a conformal mapping $\varphi$ onto the exterior of a compact disk $D$. One may assume that the disk is centered at the origin and also that $\varphi$ is normalized by the condition

$$
\varphi^{\prime}(\infty)=\lim _{z \rightarrow \infty} \frac{\varphi(z)}{z}=1 .
$$

This condition makes the mapping $\varphi$ as well as the radius $\rho$ of $D$ unique. The radius $\rho$ is called the transfinite diameter or the capacity of $S$. For its properties see Gaier [6, p. 136].

The normalized mapping $\varphi$ has an expansion of the form

$$
\varphi(z)=z+a_{0}+\frac{a_{1}}{z}+\cdots,
$$

and thus

$$
(\varphi(z))^{n}=z^{n}+f_{n, n-1} z^{n-1}+\cdots+f_{n, 0}+\frac{f_{n,-1}}{z}+\frac{f_{n,-2}}{z^{2}}+\cdots
$$

with certain coefficients $f_{n, j}, j=n-1, n-2, \ldots, 0,-1,-2, \ldots$, which could be computed from $a_{0}, a_{1}, \ldots$.

Received February 22, 1990; revised October 8, 1990 and February 15, 1991.

1991 Mathematics Subject Classification. Primary 30-04, 30C10, 30C20, 41A10, 65A05.

Key words and phrases. Faber polynomials, circular sectors, outer conformal mapping of sectors.

The second author was supported in part by NSF Grant CCR 86-19817 and by ONR Contract N00014-90-J-1599. 
The polynomial occurring in (1.3), namely

$$
F_{n}(z)=z^{n}+f_{n, n-1} z^{n-1}+\cdots+f_{n, 0},
$$

is called the $n$th Faber polynomial of $S$. These polynomials are attractive because, roughly speaking, they have good approximation power. Some estimates are given in $\S 3.3$. For more explicit properties of Faber polynomials, we refer to Gaier [7, Chapter I, §6].

The explicit construction of the Faber polynomials of a given set $S$ depends essentially on the knowledge of the conformal mapping $\varphi$. For circular sectors, Coleman and Smith [1] were able to construct the necessary conformal mapping via Schwarz-Christoffel and some other auxiliary mappings. By using a general recursion for Faber polynomials, the result of Coleman and Smith was a set of recursions which ultimately yielded the Faber polynomials. The origin of the general recursion for Faber polynomials (appearing in the sequel as Step 4 of the Coleman and Smith algorithm) is unknown to us. However, it is stated and used by Kövari and Pommerenke [9, p. 195]. A statement, including a derivation, is contained in a paper by Curtiss [3, p. 578]. The importance for numerical purposes has been stressed by Ellacott [4, p. 578].

However, this set of recursions is rather involved, so that there is hardly any chance to compute manually and explicitly the Faber polynomials for larger values of $n$. Thus, Coleman and Smith presented these polynomials explicitly only up to degree three. Beyond that, they used their recursions to compute numerically the coefficients of the Faber polynomials $F_{n}$ for $n \leq 15$ for six selected opening angles of the sector, to 14 decimals precision (Coleman and Smith [2]).

By simplifying the recursions, and by using symbolic computation, we show that it is practical to generate the Faber polynomials explicitly in terms of the opening angle to almost any degree. The symbolic computation systems we have used, and their limitations, are discussed at the end of the paper.

We also make use of another property that was at least indicated by Coleman and Smith [1, p. 238]. If we compare the coefficients that are in corresponding position in the $F_{n}$, as a function of the degree $n$, we see that they are the values of a certain polynomial. In consequence, the first terms of $F_{n}$ can be given explicitly for all $n$. We will explain this property later.

We mention another family of polynomials that are also defined with respect to the given compact set $S$, namely the Chebyshev polynomials $T_{n}$. A monic polynomial $T_{n}(z)=z^{n}+a_{n, n-1} z^{n-1}+\cdots+a_{n, 0}$ is a Chebyshev polynomial if it has minimal uniform norm on $S$ among all monic complex polynomials of the same degree. Formally, this can be stated as

$$
\left\|T_{n}\right\|_{\infty}=\max _{z \in S}\left|T_{n}(z)\right| \leq\left\|P_{n}\right\|_{\infty} \quad \text { for all } P_{n} \in \Pi_{n}
$$

where all polynomials are monic.

We will use the notation $\Pi_{n}$ for the set of all polynomials up to degree $n$ over $\mathbb{K}$, where $\mathbb{K}=\mathbb{C}$ or $\mathbb{K}=\mathbb{R}$. The definition of Chebyshev polynomials implies for the Faber polynomials $F_{n}$ that

$$
\left\|T_{n}\right\|_{\infty} \leq\left\|F_{n}\right\|_{\infty} \text {. }
$$

If $S$ is a disk or an interval, the polynomials $F_{n}$ and $T_{n}$ coincide. For the unit disk we have $F_{n}(z)=T_{n}(z)=z^{n}$ and $\left\|F_{n}\right\|_{\infty}=1$. For the interval 
$S=[0,1]$ we have

$$
F_{n}(z)=T_{n}(z)=2^{1-2 n} \cos (n \arccos (2 z-1)),
$$

and thus $\left\|F_{n}\right\|_{\infty}=2^{1-2 n}$ for $n>0$. In the latter case, the $F_{n}$ coincide with the ordinary Chebyshev polynomials adjusted to the interval $[0,1]$ and scaled so that the leading coefficient is one. We will consider how to compute the norms of Faber polynomials in $\S 3$. Further results on Faber polynomials can be found in Ellacott [5].

\section{COMPUTATION OF FABER POLYNOMIALS FOR CIRCULAR SECTORS}

Since the recursion formulae were derived by Coleman and Smith, we only describe those steps necessary to derive Faber polynomials for circular sectors

$$
S=S_{\alpha}=\{z \in \mathbb{C}:|z| \leq 1,|\arg z| \leq \alpha\},
$$

where $0 \leq \alpha \leq \pi$. The opening angle of the sector is $2 \alpha$. The transfinite diameter of $S_{\alpha}$, as given by Coleman and Smith, is

$$
\rho= \begin{cases}1 /(2-\alpha / \pi)^{2}\{(\pi / \alpha)(2-\alpha / \pi)\}^{\alpha / \pi} & \text { for } \alpha \neq 0, \\ 0.25 \text { for } \alpha=0 & \end{cases}
$$

The computational procedure for finding the Faber polynomials $F_{n}$ accounts for the geometry of $S_{\alpha}$ by means of $x$, where

$$
x=1-2 c, \quad c=\frac{\alpha}{\pi}\left(2-\frac{\alpha}{\pi}\right) .
$$

For a given $S_{\alpha}$ we do the following steps:

Step 1. Compute Legendre polynomials $P$ and polynomials $a$ :

$$
\begin{aligned}
& P_{0}(x)=1 ; \quad P_{1}(x)=x ; \\
& (n+1) P_{n+1}(x)-(2 n+1) x P_{n}(x)+n P_{n-1}(x)=0, \quad n>0 ; \\
& a_{0}=1 ; \quad a_{n}(x)=P_{n}(x)+P_{n-1}(x), \quad n>0 ;
\end{aligned}
$$

Step 2. Compute coefficients $\beta$ of the Laurent expansion:

$$
\begin{aligned}
& \beta_{0}(x)=a_{1}(x)=1+x ; \\
& (n+1) \beta_{n}(x)=a_{n+1}(x)-\sum_{k=1}^{n-1} k \beta_{k}(x) a_{n-k}(x), \quad n>0 ;
\end{aligned}
$$

Step 3. Define $b$ by multiplying $\beta$ with powers of the transfinite diameter $\rho$ :

$$
b_{n}(x)=\beta_{n}(x) \rho^{n+1}, \quad n \geq 0 ;
$$

Step 4. Compute Faber polynomials $F$ and replace $x$ by $1-2 c$ :

$$
\begin{gathered}
F_{0}(z)=1 ; \quad F_{1}(z)=z-b_{0}(x) \\
F_{n+1}(z)=\left(z-b_{0}(x)\right) F_{n}(z)-\sum_{k=1}^{n-1} b_{k}(x) F_{n-k}(z) \\
-(1+n) b_{n}(x), \quad n>0 .
\end{gathered}
$$


Observe that $c$ increases from 0 to 1 and $\rho$ increases from 0.25 to 1 as $\alpha$ increases from 0 to $\pi$. Apart from small notational differences, Coleman and Smith's computation is the same. However, we can make some simplifications. When $\alpha=\pi$, the $n$th Faber polynomial reduces to $z^{n}$, which is reflected by $c-1=0$, or, equivalently, by $x+1=0$. Therefore, the Faber polynomial $F_{n}$ must have the form $F_{n}(z)=z^{n}+(1-c) \pi_{n-1}(z)$, where $\pi_{n-1} \in \Pi_{n-1}$. Furthermore, we can eliminate the transfinite diameter $\rho$ by observing that

$$
\begin{aligned}
F_{n}(z \rho)=\rho^{n}\left(z^{n}+(1-c)\left(p_{n-1,0} z^{n-1}\right.\right. & +p_{n-1,1} z^{n-2} \\
& \left.\left.+\cdots+p_{n-1, n-1}\right)\right), \quad n>0 .
\end{aligned}
$$

This follows from Steps 3 and 4. We use the following notation:

$$
\begin{aligned}
& \Phi_{0}=1 ; \\
& \Phi_{n}(z)=z^{n}+(1-c)\left(p_{n-1,0} z^{n-1}+p_{n-1,1} z^{n-2}+\cdots+p_{n-1, n-1}\right), \\
& n>0,
\end{aligned}
$$

where $\Phi_{n}$ is now independent of $\rho$ and the coefficients $p_{n-1, j} \in \Pi_{j}, j=$ $0,1, \ldots, n-1$, are polynomials in the variable $c$. The geometric meaning of $c$ is given in (2.3). The polynomials

$$
\widetilde{F}_{n}(z)=F_{n}(z) \rho^{-n}=\Phi_{n}(z / \rho)
$$

are the scaled Faber polynomials.

Now it is easy to see that the functions $\Phi_{n}$ obey the same recursion as the functions $F_{n}$ in Step 4, after the $b$ 's are replaced with the $\beta$ 's. Thus, computing $\Phi_{n}$ rather than $F_{n}$ eliminates Step 3. But we can do better. Since the polynomial factor of $1-c$ in (2.5) contains already all the essential information about $\Phi_{n}$, we derive a recursion for this factor. For $c<1$, we denote the factor by

$$
\phi_{n-1}(z)=p_{n-1,0} z^{n-1}+p_{n-1,1} z^{n-2}+\cdots+p_{n-1, n-1}, \quad n>0,
$$

which changes $(2.5)$ to

$$
\Phi_{0}=1 ; \quad \Phi_{n}(z)=z^{n}+(1-c) \phi_{n-1}(z), \quad n>0 .
$$

In terms of the newly introduced $\phi_{n-1}$, the recursion of Step 4 reads:

$$
\begin{gathered}
\phi_{0}=-2 ; \quad \tilde{\beta}_{k}=\beta_{k} /(1-c), \\
\phi_{n}=\left(z-\beta_{0}\right) \phi_{n-1}-\sum_{k=1}^{n-1} \beta_{k} \phi_{n-k-1} \\
-\sum_{k=0}^{n-1} \tilde{\beta}_{k} z^{n-k}-(n+1) \tilde{\beta}_{n}, \quad n>0 .
\end{gathered}
$$

Since $a_{n}(-1)=P_{n}(-1)+P_{n-1}(-1)=0$, where $n>0$, all polynomials $a$ have the factor $x+1$, or, equivalently, the factor $1-c$. The same applies to the polynomials $\beta_{n}$, as is evident from Step 2. Therefore, by one sweep of the Horner scheme at $x=-1$ or at $c=1$, this factor can be cancelled, thus obtaining $\tilde{\beta}_{k}=\beta_{k} /(1-c)$.

It is unreasonable to work with the two closely related geometric quantities $x$ and $c$ simultaneously. Therefore, we delete $x$ and work with $c$ only. Since the 
Legendre polynomials are only used to generate the polynomials $a$, they need not be stored. In the following algorithm we assume that we want to compute all Faber polynomials up to a certain degree $n_{\max }>1$. We replace the above steps by

Algorithm. Apply simplifications, compute $\phi_{n}$ :

A. Initialization:

$P 0=1 ; P 1=1-2 c ; \phi[0]=-2$;

$a[1]=2-2 c ; \beta[0]=2-2 c ; \tilde{\beta}[0]=2$;

B. For $n=1$ to $n_{\max }-1$ compute

$P 2=((2 n+1)(1-2 c) P 1-n P 0) /(n+1)$;

$P 0=P 1 ; P 1=P 2$

$a[n+1]=P 0+P 1$

$\beta[n]=\left(a[n+1]-\sum_{k=1}^{n-1} k \beta[k] a[n-k]\right) /(n+1) ; \tilde{\beta}[n]=\beta[n] /(1-c) ;$

$\phi[n]=z \phi[n-1]-(n+1) \tilde{\beta}[n]-\sum_{k=0}^{n-1}\left(\beta[k] \phi[n-k-1]+\tilde{\beta}[k] z^{n-k}\right) ;$

print polynomial coefficients of $\phi[n]$; measure the computing time;

$\Phi[n+1]=z^{n+1}+(1-c) \phi[n] ;\{$ may be deleted

$F[n+1](z)=\rho^{n+1} \Phi[n+1](z / \rho) .\{$ may be deleted $\}$

Example. We compute the quantities necessary to generate the Faber polynomials $F_{n}$ and the polynomials $\phi_{n-1}$ for $n \leq 4$. We do not list the $\Phi_{n}$, since they are immediate from $\phi_{n-1}$ and $\left(2.5^{\prime}\right)$.

Step 1. $P_{0}=1 ; P_{1}(x)=x ; P_{2}(x)=\frac{1}{2}\left(-1+3 x^{2}\right) ; P_{3}(x)=\frac{1}{2}\left(-3 x+5 x^{3}\right)$; $P_{4}(x)=\frac{1}{8}\left(3-30 x^{2}+35 x^{4}\right) ; a_{0}=1 ; a_{1}(x)=1+x ; a_{2}(x)=\frac{1}{2}\left(-1+2 x+3 x^{2}\right)$; $a_{3}(x)=\frac{1}{2}\left(-1-3 x+3 x^{2}+5 x^{3}\right) ; a_{4}(x)=\frac{1}{8}\left(3-12 x-30 x^{2}+20 x^{3}+35 x^{4}\right)$;

Step 2. $\beta_{0}(x)=1+x ; \beta_{1}(x)=\frac{1}{4}\left(-1+2 x+3 x^{2}\right) ; \beta_{2}(x)=\frac{1}{12}\left(-1-7 x+x^{2}+7 x^{3}\right)$; $\beta_{3}(x)=\frac{1}{48}\left(5+4 x-30 x^{2}-4 x^{3}+25 x^{4}\right)$;

Step 3. $b_{n}=\rho^{n+1} \beta_{n}$;

Step 4. $F_{0}=1 ; F_{1}(z)=z-\rho(1+x) ; F_{2}(z)=z^{2}-2 \rho(1+x) z+\frac{1}{2} \rho^{2}\left(3+2 x-x^{2}\right)$; $F_{3}(z)=z^{3}-3 \rho(1+x) z^{2}+\frac{3}{4} \rho^{2}\left(5+6 x+x^{2}\right) z-\frac{1}{2} \rho^{3}\left(3+x-x^{2}+x^{3}\right) ; F_{4}(z)$ $=z^{4}-4 \rho(1+x) z^{3}+\rho^{2}\left(7+10 x+3 x^{2}\right) z^{2}-\frac{1}{3} \rho^{3}\left(17+23 x+7 x^{2}+x^{3}\right) z+$ $\frac{1}{8} \rho^{4}\left(11+4 x+2 x^{2}+4 x^{3}-5 x^{4}\right)$.

Algorithm. $a_{1}(c)=2-2 c ; a_{2}(c)=2-8 c+6 c^{2} ; a_{3}(c)=2-18 c+36 c^{2}-20 c^{3}$; $a_{4}(c)=2-32 c+120 c^{2}-160 c^{3}+70 c^{4}$

$\tilde{\beta}_{0}=2 ; \quad \tilde{\beta}_{1}(c)=1-3 c ; \quad \tilde{\beta}_{2}(c)=-8 / 3 c+14 / 3 c^{2} ; \quad \tilde{\beta}_{3}(c)=-4 / 3 c+$ $23 / 3 c^{2}-25 / 3 c^{3}$

$\phi_{0}=-2 ; \phi_{1}(z)=-4 z+2(1+c) ; \phi_{2}(z)=-6 z^{2}+3(3-c) z-2\left(1+2 c^{2}\right) ;$

$\phi_{3}(z)=-8 z^{3}+4(5-3 c) z^{2}+\left(-16+\frac{32}{3} c-\frac{8}{3} c^{2}\right) z+2\left(1+c-3 c^{2}+5 c^{3}\right)$.

In order to make the structure of the coefficients $p_{n-1, j}$ defined in (2.5) more explicit, we show their dependence on $n$. For $n \geq 0$, for $j=0,1, \ldots, n$, and for $s=j(j+1) / 2+1$, we define

$$
p_{n, j}(c)=\gamma_{n+1, s}+\gamma_{n+1, s+1} c+\cdots+\gamma_{n+1, s+j} c^{j} .
$$


Therefore, the coefficients $\gamma_{n+1, k}$ may be regarded as the elements in row $n+1$ of a matrix

$$
\Gamma=\left(\gamma_{j k}\right), \quad j=1,2, \ldots ; k=1,2, \ldots, j(j+1) / 2,
$$

which has infinitely many rows. Row $j$ is finite with $j(j+1) / 2$ elements. As an example, we put the above $\phi_{3}$ into the form of notation (2.9) and obtain

$$
\begin{aligned}
\phi_{3}(z)= & -8 z^{3}+4(5-3 c) z^{2}+\left(-16+\frac{32}{3} c-\frac{8}{3} c^{2}\right) z+2\left(1+c-3 c^{2}+5 c^{3}\right) \\
= & p_{30}(c) z^{3}+p_{31}(c) z^{2}+p_{32}(c) z+p_{33} \\
= & \gamma_{41} z^{3}+\left(\gamma_{42}+\gamma_{43} c\right) z^{2}+\left(\gamma_{44}+\gamma_{45} c+\gamma_{46} c^{2}\right) z \\
& +\gamma_{47}+\gamma_{48} c+\gamma_{49} c^{2}+\gamma_{4,10} c^{3} .
\end{aligned}
$$

We denote the submatrix of $\Gamma$ consisting of the first $n$ rows by

$$
\Gamma_{n}=\left(\gamma_{j k}\right), \quad j=1,2, \ldots, n ; k=1,2, \ldots, j(j+1) / 2 .
$$

Theorem 1. All entries of the matrix $\Gamma$ are rational.

Proof. Immediate from Steps 1-4 and (2.4), (2.9).

In Table 5 of the appendix (see supplement section at the end of this issue), we list the polynomials $p_{n-1, j}$ for $j=0(1) n-1$ and $n=1(1) 20$. Compare also (2.9). The polynomials $p_{n-1, j}$ define the polynomials $\phi_{n-1}(z)=$ $\sum_{j=0}^{n-1} p_{n-1, j} z^{n-j-1}$. The polynomials $\phi_{n-1}(z)$ define the polynomials $\Phi_{n}(z)$ (compare (2.5)). In turn, the polynomials $\Phi_{n}(z)$ define the Faber polynomials $F_{n}(z)$ (compare (2.6) and (2.2)).

If we were to store all coefficients for degrees up to $n$, we would need $1+3+$ $6+\cdots+0.5 n(n+1)=n(n+1)(n+2) / 6$ storage locations. For $n=5,10,15,20$, and 100 , these numbers are $35,220,680,1540$, and 171700 , respectively. In comparison, Coleman and Smith [2] required 628 numerical coefficients for the coefficients of the Faber polynomials up to degree 15 on only six selected opening angles.

From Table 5 of the appendix we have

$$
\Gamma_{10}=\left(\begin{array}{ccccccccccc}
-2 & & & & & & & & & & \\
-4 & 2 & 2 & & & & & & & & \\
-6 & 9 & -3 & -2 & 0 & -4 & & & & & \\
-8 & 20 & -12 & -16 & \frac{32}{3} & -\frac{8}{3} & 2 & 2 & -6 & 10 & \\
-10 & 35 & -25 & -50 & \frac{160}{3} & -\frac{40}{3} & 25 & -\frac{65}{3} & \frac{10}{3} & \frac{10}{3} & \ldots \\
-12 & 54 & -42 & -112 & 144 & -44 & 105 & -151 & 65 & -7 & \ldots \\
-14 & 77 & -63 & -210 & \frac{896}{3} & \frac{-308}{3} & 294 & -518 & 287 & -49 & \ldots \\
-16 & 104 & -88 & -352 & \frac{1600}{3} & -\frac{592}{3} & 660 & -\frac{3908}{3} & \frac{2476}{3} & \frac{-500}{3} & \ldots \\
-18 & 135 & -117 & -546 & 864 & -336 & 1287 & -2733 & 1884 & -420 & \ldots \\
-20 & 170 & -150 & -800 & \frac{3920}{3} & -\frac{1580}{3} & 2275 & -5085 & 3715 & -885 & \ldots
\end{array}\right) .
$$


After some calculation, we see that

$$
\begin{aligned}
\gamma_{n 1}= & -2-2(n-1)=-2 n \\
\gamma_{n 2}= & 2+7(n-2)+2(n-2)(n-3) \\
\gamma_{n 3}= & 2-5(n-2)-2(n-2)(n-3) \\
\gamma_{n 4}= & -2-14(n-3)-10(n-3)(n-4)-\frac{4}{3}(n-3)(n-4)(n-5) \\
\gamma_{n 5}= & \frac{1}{3}(32(n-3)+48(n-3)(n-4)+8(n-3)(n-4)(n-5)) \\
\gamma_{n 6}= & -4+\frac{4}{3}(n-3)-6(n-3)(n-4)-\frac{4}{3}(n-3)(n-4)(n-5) \\
\gamma_{n 7}= & 2+23(n-4)+28.5(n-4)(n-5)+\frac{26}{3}(n-4)(n-5)(n-6) \\
& +\frac{2}{3}(n-4)(n-5)(n-6)(n-7) ; \\
\gamma_{n 8}= & 2-\frac{71}{3}(n-4)-\frac{317}{6}(n-4)(n-5)-22(n-4)(n-5)(n-6) \\
& -2(n-4)(n-5)(n-6)(n-7) ; \\
\gamma_{n 9}= & -6+\frac{28}{3}(n-4)+\frac{157}{6}(n-4)(n-5)+18(n-4)(n-5)(n-6) \\
& +2(n-4)(n-5)(n-6)(n-7) ; \\
\gamma_{n 10}= & 10-\frac{20}{3}(n-4)-\frac{11}{6}(n-4)(n-5)-\frac{14}{3}(n-4)(n-5)(n-6) \\
& -\frac{2}{3}(n-4)(n-5)(n-6)(n-7)
\end{aligned}
$$

is valid for all $n$. This implies that we know the entire column of $\Gamma$ as soon as the first entries are known.

The regularity of the above matrix $\Gamma$ is expressed in the following theorem.

Theorem 2. Let $\Gamma=\left(\gamma_{j k}\right)$ be the matrix defined in (2.9), (2.10), (2.4), (2.5). For each fuxed $k=1,2, \ldots$, there exists a polynomial $\pi_{k} \in \Pi_{j_{0}}$ such that

$$
\gamma_{j k}=\pi_{k}(j) \text { for all } j \geq j_{0},
$$

where $j_{0}$ is uniquely defined by

$$
j_{0}\left(j_{0}+1\right) / 2<k \leq\left(j_{0}+1\right)\left(j_{0}+2\right) / 2 .
$$

Proof. By the recursion (2.8), using induction.

The above $\gamma_{j k}$ are just the coefficients of the polynomial $p_{j-1, j_{0}} \in \Pi_{j_{0}}$. In order to compute the $\pi_{k}$, we interpolate the first entries of column $k$ of $\Gamma$. That is, we interpolate the points $\left(j, \gamma_{j k}\right)$ for $j=j_{0}, j_{0}+1, \ldots, 2 j_{0}$. If we choose the Newton form for $\pi_{k}$, then the result can be put in the form

$$
\begin{aligned}
\pi_{k}(n)= & d_{0 k}+d_{1 k}\left(n-j_{0}\right)+d_{2 k}\left(n-j_{0}\right)\left(n-j_{0}-1\right) \\
& +\cdots+d_{j_{0} k}\left(n-j_{0}\right)\left(n-j_{0}-1\right) \cdots\left(n-2 j_{0}+1\right),
\end{aligned}
$$

and we can store these results in a matrix

$$
D=\left(d_{j k}\right), \quad k=1,2, \ldots ; j=0,1, \ldots, j_{0},
$$

where $j_{0}$ was already defined in (2.14). Examples were already given directly after matrix (2.12).

Suppose we know the first $n$ Faber polynomials, or, equivalently, the matrix $\Gamma_{n}$. By Theorem 2, the first $[n / 2]([n / 2]+1) / 2$ columns of the infinite matrix $\Gamma$ are known completely. Here, $[x]$ denotes the largest integer less than or equal 
to $x$. In particular, Theorem 2 gives full information about the growth of the coefficients of the Faber polynomials.

\section{TWO-NORMS AND INFINITY NORM OF FABER POLYNOMIALS ON CIRCULAR SECTORS}

We now consider $\left\|F_{n}\right\|_{\infty}$ and $\left\|F_{n}\right\|_{2}$. Note that the latter norm can be explicitly computed. In the case of the two-norm, we will consider the area norm and the line norm separately. We also state a conjecture about the uniform norm that would allow an explicit and easy computation of $\left\|F_{n}\right\|_{\infty}$.

3.1. The area norm of Faber polynomials on circular sectors. In order to compute the (area) two-norm, assume first that the opening angle $\alpha$ of the sector $S_{\alpha}$ is positive. In this case, the square of the two-norm of the scaled Faber polynomials (cf. (2.6)) is

$$
\left\|\widetilde{F}_{n}\right\|_{2}^{2}=\int_{S_{\alpha}}\left|\Phi_{n}(z / \rho)\right|^{2} d x d y=\rho^{2} \int_{S_{\alpha} / \rho}\left|\Phi_{n}(u)\right|^{2} d v d w,
$$

where $z=x+i y, u=v+i w$, and $S_{\alpha} / \rho$ is the sector with radius $1 / \rho$ and opening angle $2 \alpha$. Using $\left(2.5^{\prime}\right)$ and (2.7), we have

$$
\begin{aligned}
\left|\Phi_{n}(u)\right|^{2} & =\left|u^{n}+(1-c) \phi_{n-1}(u)\right|^{2} \\
& =|u|^{2 n}+2(1-c) \Re\left\{\bar{u}^{n} \phi_{n-1}(u)\right\}+(1-c)^{2}\left|\phi_{n-1}(u)\right|^{2},
\end{aligned}
$$

where the overbar denotes complex conjugation and $\mathfrak{R}$ denotes the real part of the corresponding complex number. Thus, the problem is reduced to integrating terms of the form $\mathfrak{R} u^{s} \bar{u}^{t}, s, t \geq 0$. Using polar coordinates, we obtain

$$
I_{s, t}=\rho^{2} \int_{S_{\alpha} / \rho} \mathfrak{R} u^{s} \bar{u}^{t} d v d w= \begin{cases}\frac{2 \rho^{-s-t} \sin (s-t) \alpha}{(s-t)(s+t+2)} & \text { for } s \neq t, \\ \frac{\rho^{-2 s} \alpha}{s+1} & \text { for } s=t .\end{cases}
$$

Combining these results, we obtain for $\alpha>0$ the following final formula:

$$
\begin{aligned}
\left\|\widetilde{F}_{n}\right\|_{2}^{2}= & I_{n, n}+2(1-c) \sum_{j=0}^{n-1} p_{n-1, j} I_{n-1-j, n} \\
& +(1-c)^{2} \sum_{j=0}^{n-1} p_{n-1, j}^{2} I_{n-1-j, n-1-j} \\
& +2(1-c)^{2} \sum_{j>k}^{n-1} p_{n-1, j} p_{n-1, k} I_{n-1-j, n-1-k} .
\end{aligned}
$$

In the disk case $(\alpha=\pi, c=\rho=1)$, this formula reduces to

$$
\left\|\widetilde{F}_{n}\right\|_{2}^{2}=\left\|F_{n}\right\|_{2}^{2}=\frac{\pi}{n+1} .
$$

In the interval case $\left(\alpha=c=0, \rho=\frac{1}{4}\right)$ we can use the explicit form of the $F_{n}$ given at the end of $\S 1$. The square of the two-norm of the scaled Faber polynomials is then

$$
\left\|\widetilde{F}_{n}\right\|_{2}^{2}=4 \int_{0}^{1} \cos ^{2}\{n \arccos (2 x-1)\} d x=2-\frac{2}{4 n^{2}-1} .
$$


3.2. The line norm of Faber polynomials on circular sectors. The square of the line norm of the scaled Faber polynomials is defined by

$$
\hat{\|} \widetilde{F}_{n} \hat{\|}_{2}^{2}=\int_{\partial S_{\alpha}}\left|\Phi_{n}(z / \rho)\right|^{2} d s=\rho \int_{\partial S_{\alpha} / \rho}\left|\Phi_{n}(u)\right|^{2} d \sigma
$$

where $s$ is the line element in the $z$-plane, $\sigma$ is the line element in the $u$ plane, and $\partial S$ is the boundary of $S$. With these conventions, the results of the previous subsection apply essentially unchanged, after replacing the area integrals $I_{s, t}$ given in (3.3) with the corresponding line integrals. For $0<\alpha<\pi$ these integrals are

$$
\begin{aligned}
\widehat{I}_{s, t} & =\rho \int_{\partial S_{\alpha} / \rho} \mathfrak{R} u^{s} \bar{u}^{t} d \sigma \\
& = \begin{cases}2 \rho^{-s-t}\left\{\frac{\cos (s-t) \alpha}{(s+t+1)}+\frac{\sin (s-t) \alpha}{s-t}\right\} & \text { for } s \neq t, \\
2 \rho^{-2 s}\left\{\frac{1}{2 s+1}+\alpha\right\} & \text { for } s=t .\end{cases}
\end{aligned}
$$

For $\alpha=0$ we delete the factor 2 , and for $\alpha=\pi$ we omit the integrals over the straight parts of the sector. So, we obtain

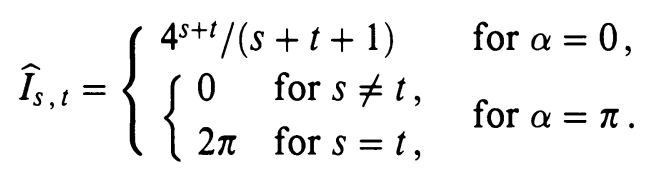

Thus, the final formula for $\| \widetilde{F}_{n} \hat{\|}_{2}^{2}$ is the same as formula (3.4), when replacing $I_{s, t}$ with $\widehat{I}_{s, t}$ from $(3.8 \mathrm{a})$ and $(3.8 \mathrm{~b})$.

3.3. Infinity norm of Faber polynomials on circular sectors. The results of Coleman and Smith [1], partly due to Pommerenke [10] and Walsh [12, p. 319] are:

$$
\begin{gathered}
\left\|T_{n}\right\|_{\infty} \leq\left\|F_{n}\right\|_{\infty} \leq 2\left\|T_{n}\right\|_{\infty}, \\
1 \leq\left\|\widetilde{F}_{n}\right\|_{\infty} \leq 2, \\
\lim _{n \rightarrow \infty}\left\|\widetilde{F}_{n}\right\|_{\infty}= \begin{cases}1 & \text { for } \alpha=\pi, \\
2(1-\alpha / \pi) & \text { for } 0 \leq \alpha \leq \pi / 4,\end{cases}
\end{gathered}
$$

where $\widetilde{F}_{n}$ are the scaled Faber polynomials as introduced in (2.6).

The situation for obtaining explicit expressions for $\left\|F_{n}\right\|_{\infty}$ is not so favorable. However, supported by numerical experiments, we formulate the following conjecture.

Conjecture. Assume that $0<\alpha<\pi$ and $n>0$. Then the norm $\left\|F_{n}\right\|_{\infty}$ is always attained at the origin $z=0$ or at the two corners $z=e^{ \pm i \alpha}$ of the sector $S_{\alpha}$. For fixed $n$ there is a critical angle $\alpha_{n}$ such that for $\alpha<\alpha_{n}$ the norm is attained only at the origin, and for $\alpha>\alpha_{n}$ the norm is attained only at the two corners. For $\alpha=\alpha_{n}$ the norm is attained only at the origin and at the two corners.

For $n=1$ we can find the above critical angle by solving $\left|F_{1}(0)\right|=F_{1}\left(e^{i \alpha}\right) \mid$ for $\alpha$ with a pocket calculator (HP 15C) and obtain as solution $\alpha_{1} \approx 0.61838434$ 
TABLE 1

Critical angles for Faber polynomials $F_{n}$ on sectors

\begin{tabular}{rcc}
\hline$n$ & alpha(deg) & alpha \\
\hline 1 & 35.430813 & 0.61838434 \\
2 & 59.059789 & 1.03078777 \\
3 & 43.672788 & 0.76223395 \\
4 & 46.840804 & 0.81752625 \\
5 & 44.221977 & 0.77181909 \\
6 & 47.007470 & 0.82043512 \\
7 & 45.619633 & 0.79621279 \\
8 & 43.976473 & 0.76753425 \\
9 & 45.575804 & 0.79544783 \\
10 & 45.511724 & 0.79432944 \\
11 & 45.399940 & 0.79237843 \\
12 & 44.403878 & 0.77499388 \\
13 & 45.732598 & 0.79818441 \\
14 & 45.099206 & 0.78712963 \\
15 & 45.112012 & 0.78735314 \\
16 & 44.837307 & 0.78255863 \\
17 & 45.535540 & 0.79474510 \\
18 & 45.097395 & 0.78709802 \\
19 & 44.829223 & 0.78241755 \\
20 & 45.129013 & 0.78764986 \\
\hline & &
\end{tabular}

$\approx 35.430813^{\circ}$. According to our numerical tests, the critical angles for $3 \leq$ $n \leq 20$ are all close to, but not equal to, $\pi / 4$. For $n=2$, the critical angle is approximately $60^{\circ}$. The computed values are shown in Table 1.

In geometric terms, the above conjecture means that the contour line (or lemniscate) $L=\left\{z \in \mathbb{C}:\left|F_{n}(z)\right|=\left\|F_{n}\right\|_{\infty}\right\}$ contains the underlying sector $S_{\alpha}$ in its interior and touches it at most at the corners of the sector. A graph for $\alpha=\pi / 4, n=4$ is given in Figure 1 . It was produced with MATLAB from data computed by a Pascal program. Generally, these contour lines approach the sector $S_{\alpha}$ with increasing degree $n$. A similar graph for a Chebyshev polynomial is given by Grothkopf and Opfer [8]. Some selected values of $\left\|\widetilde{F}_{10}\right\|$ for all considered norms are shown in Table 2.

An upper bound for the two-norms is easily obtained by means of infinity norms. If $p_{n}$ is any polynomial in $\Pi_{n}$ on the sector $S_{\alpha}$, we have for $\alpha>0$

$$
\begin{aligned}
& \left\|p_{n}\right\|_{2} \leq \sqrt{\alpha}\left\|p_{n}\right\|_{\infty}, \quad \hat{\|} p_{n} \hat{\|}_{2} \leq \hat{c}\left\|p_{n}\right\|_{\infty}, \\
& \hat{c}= \begin{cases}\sqrt{2+2 \alpha} & \text { for } 0<\alpha<\pi, \\
\sqrt{2 \pi} & \text { for } \alpha=\pi .\end{cases}
\end{aligned}
$$




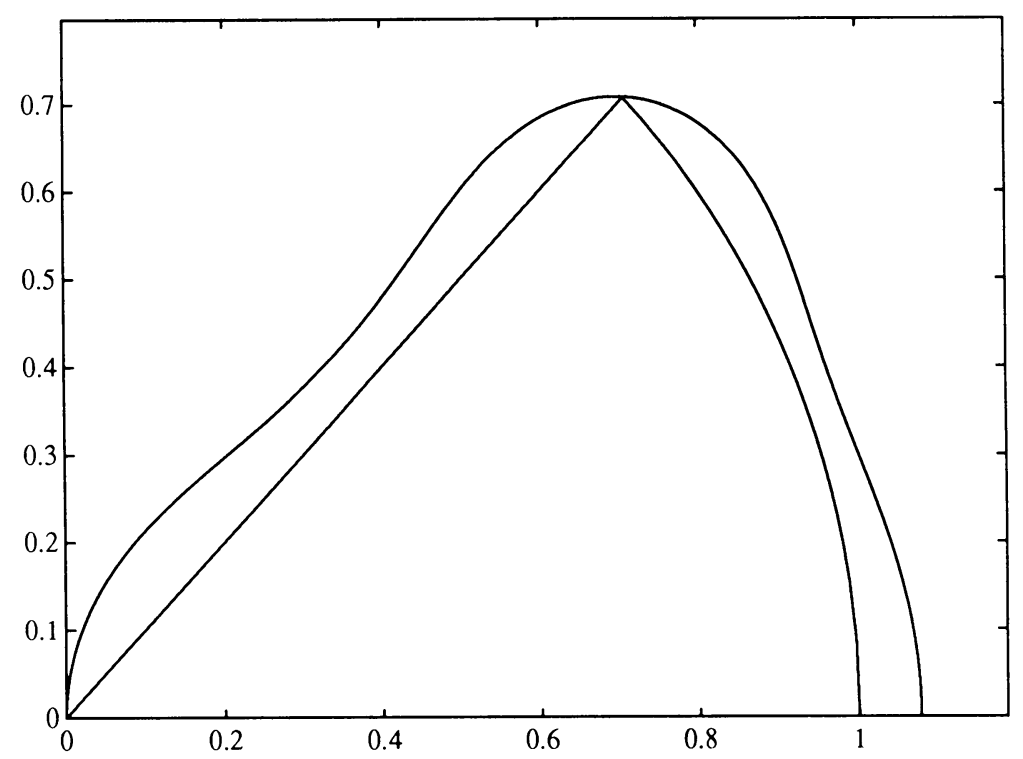

FIGURE 1

Lemniscate of Faber polynomial $F_{4}$ on quarter disk (upper half)

TABLE 2

Norms of $\widetilde{F}_{10}$ for selected opening angles

\begin{tabular}{cccc}
\hline Angle(deg) & Area norm & Line norm & Max norm \\
\hline 0 & 1.41244 & 1.41244 & 2.00000 \\
1 & 0.14464 & 1.73706 & 1.99486 \\
2 & 0.17507 & 1.63409 & 1.98289 \\
5 & 0.20528 & 1.54936 & 1.93763 \\
10 & 0.22675 & 1.56115 & 1.88580 \\
15 & 0.24195 & 1.60252 & 1.84438 \\
30 & 0.28744 & 1.75067 & 1.65220 \\
45 & 0.33064 & 1.89025 & 1.51534 \\
60 & 0.37649 & 2.02449 & 1.49257 \\
90 & 0.47260 & 2.26905 & 1.49433 \\
120 & 0.62861 & 2.48243 & 1.47365 \\
135 & 0.72261 & 2.57852 & 1.38143 \\
150 & 0.71351 & 2.60358 & 1.68426 \\
165 & 0.55964 & 2.52959 & 1.68443 \\
170 & 0.53384 & 2.51431 & 1.43410 \\
175 & 0.52961 & 2.51008 & 1.14367 \\
178 & 0.53194 & 2.51550 & 1.02539 \\
179 & 0.53307 & 2.51966 & 1.00645 \\
180 & 0.53441 & 2.50663 & 1.00000 \\
\hline & & &
\end{tabular}




\section{REPORT ON COMPUTATIONS}

We used several computers and symbolic algebra systems, as listed in Table 3. The SIEMENS 7.882 computer is a large mainframe, the Symbolics 3650 is a workstation whose native language is Lisp, and the other computers are personal microcomputers.

We tested our algorithms on the various machines by computing the Faber polynomials up to a certain degree $n$ and observing the corresponding computing times. Denote by time ${ }_{n}$ the computing time for computing all Faber polynomials up to degree $n$ for a fixed combination of computer and program. We observed in our experiments that the quotient $q_{n}=$ time $_{n} /$ time $_{n-1}$ depended on the specific computer and program, but was almost independent of $n$. Thus, the computing time can be predicted by the formula

$$
\text { time }_{n}=\left(q_{n_{0}}\right)^{n-n_{0}} \text { time }_{n_{0}}, \quad n \geq n_{0} .
$$

Table 4 summarizes our observations.

TABLE 3

List of computers and languages used

\begin{tabular}{lll}
\hline \multicolumn{2}{l}{ Case No. Computer } & Language \\
\hline I & SIEMENS 7.882 & REDUCE 3.3 \\
& & CRand Corporation \\
\hline II & IBM PS/2 Modell 70 A21 & REDUCE 3.3 \\
& & CNorthwest Computer Algorithms \\
\hline III & Symbolics 3650 & MACSYMA 414.62 \\
& & CSymbolics \\
\hline IV & IBM PS/2 Modell 70 A21 MATHEMATICA & @1988 Wolfram Research \\
& & RIEMANN 1.b \\
\hline V & ATARI MEGA ST4 & C1989 Jörg Begemann \\
& & und Alexander Niemeyer \\
\hline
\end{tabular}

TABLE 4

Behavior of computer/programsystem with respect to algorithm

\begin{tabular}{llllll}
\hline & I & II & III & IV & V \\
\hline time for $n=10$ & $2.7^{\prime}$ & $\dagger$ & $19^{\prime \prime}$ & $9^{\prime} 25^{\prime \prime}$ & $9^{\prime} 49^{\prime \prime}$ \\
\hline last $n$ & 20 & 9 & 20 & 15 & 20 \\
\hline time for last $n$ & $18.0^{\prime}$ & $1.5^{\prime} \ddagger$ & $7.4^{\prime}$ & $22 \mathrm{~h} 50^{\prime}$ & $10 \mathrm{~h} 58^{\prime}$ \\
\hline time $_{n} /$ time $_{n-1}$ & 1.22 & 1.50 & 1.63 & 2.69 & 1.46 \\
\hline
\end{tabular}

${ }^{\dagger}$ Not available because of memory overflow.

$\ddagger$ Most likely wrongly reported by REDUCE. 


\section{ACKNOWLEDGMENT}

We would like to thank Günther Jantzen for conducting the experiments using Mathematica.

\section{BIBLIOGRAPHY}

1. John P. Coleman and Russel A. Smith, The Faber polynomials for circular sectors, Math. Comp. 49 (1987), 231-241.

2. __ Supplement to the Faber polynomials for circular sectors, Math. Comp. 49 (1987), S1-S4.

3. J. H. Curtiss, Faber polynomials and the Faber series, Amer. Math. Monthly 78 (1971), 577-596.

4. S. W. Ellacott, Computation of Faber series with application to numerical polynomial approximation in the complex plane, Math. Comp. 40 (1983), 575-587.

5. __ On Faber polynomials and Chebyshev polynomials, Approximation Theory (C. K. Chui, L. L. Schumaker, and J. D. Ward, eds.), Academic Press, 1983, pp. 457-464.

6. Dieter Gaier, Konstruktive Methoden der konformen Abbildung, Springer, Berlin, Göttingen, Heidelberg, 1964.

7. _ Lectures on complex approximation, Birkhäuser, Boston, Basel, Stuttgart, 1987.

8. Uwe Grothkopf and Gerhard Opfer, Complex Chebyshev polynomials on circular sectors with degree six or less, Math. Comp. 33 (1982), 111-120.

9. T. Kövari and Christian Pommerenke, On Faber polynomials and Faber expansions, Math. Z. 99 (1967), 193-206.

10. Christian Pommerenke, Über die Faberschen Polynome schlichter Funktionen, Math. Z. 85 (1964), 197-208.

11. Gerhard Rayna, REDUCE, software for algebraic computation, Springer, New York, 1987.

12. Joseph L. Walsh, Interpolation and approximation by rational functions in the complex domain, 5th ed., Amer. Math. Soc., Providence, RI, 1969.

13. Stephen Wolfram, Mathematica-a system for doing mathematics by computer, AddisonWesley, Redwood City, CA, 1988.

Konrad-Zuse-Zentrum für Informationstechnik Berlin, Heilbronnerstrasse 10, DW1000 BERLIN 31, GERMANY

Department of Computer Sciences, Purdue University, West Lafayette, Indiana 47907

Institut für ANGewandte Mathematik, Universität Hamburg, Bundesstrasse 55, DW2000 HAMBURG 13, GERMANY 


\section{Supplement to}

EXPLICIT FABER POLYNOMIALS

ON CIRCULAR SECTORS

\section{KARIN GATERMANN, CHRISTOPH HOFFMANN, AND GERHARD OPFER}

\section{Appendix}

Table 5. Faber polynomials up to degree 20

Polynomials $\phi_{n-1}=\sum_{j=0}^{n-1} p_{j} z^{n-j-1}$ for $1 \leq n \leq 20$, cf. $(2.7),(2.9)$

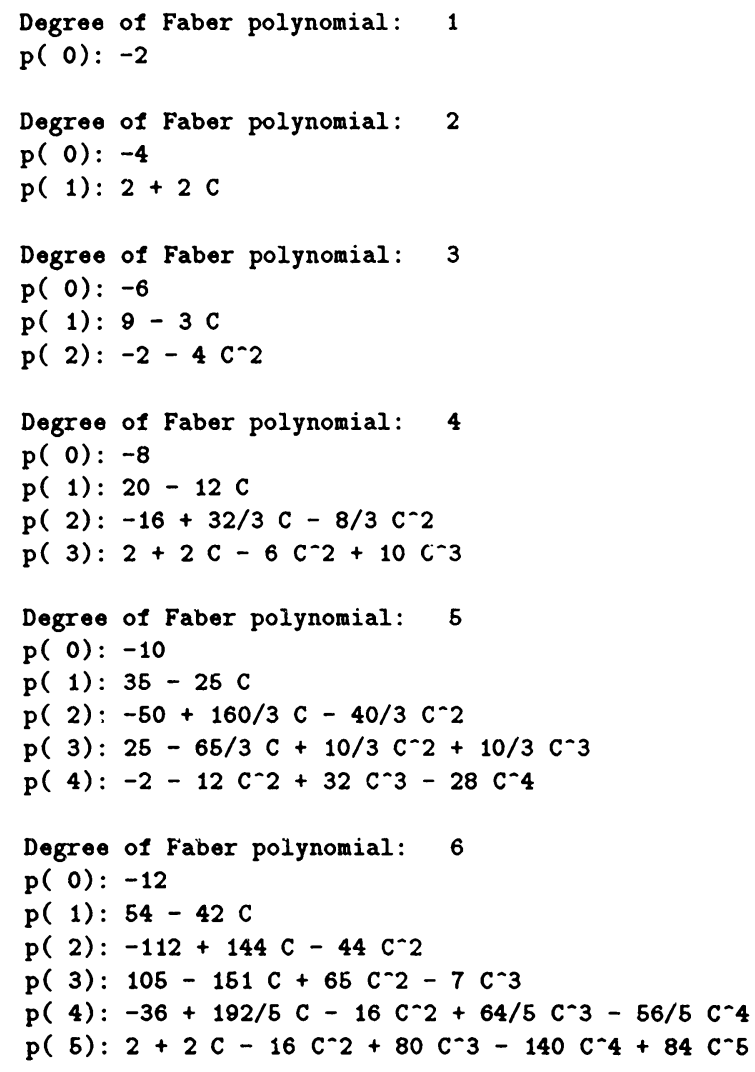



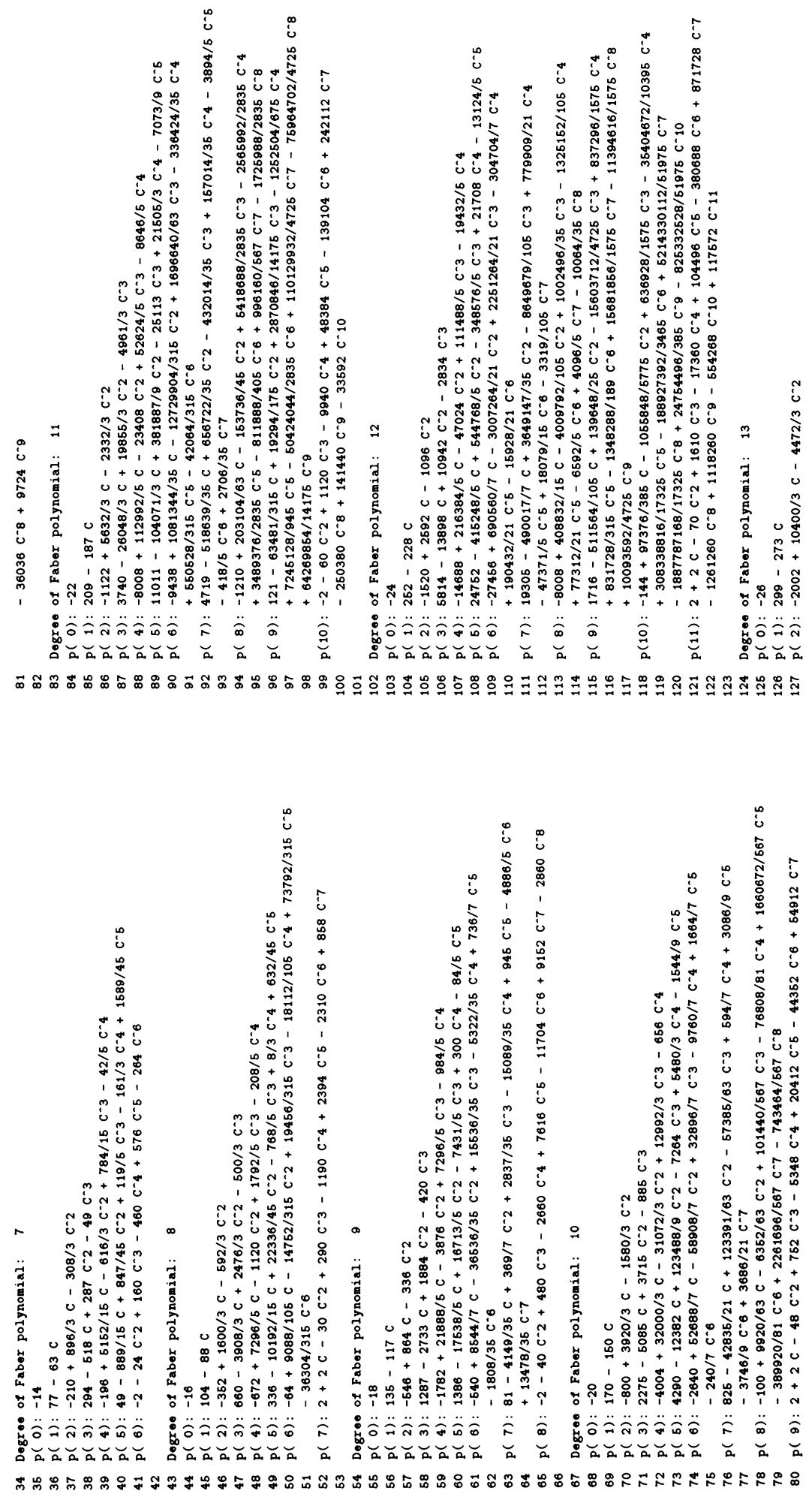


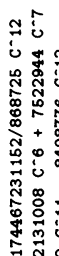

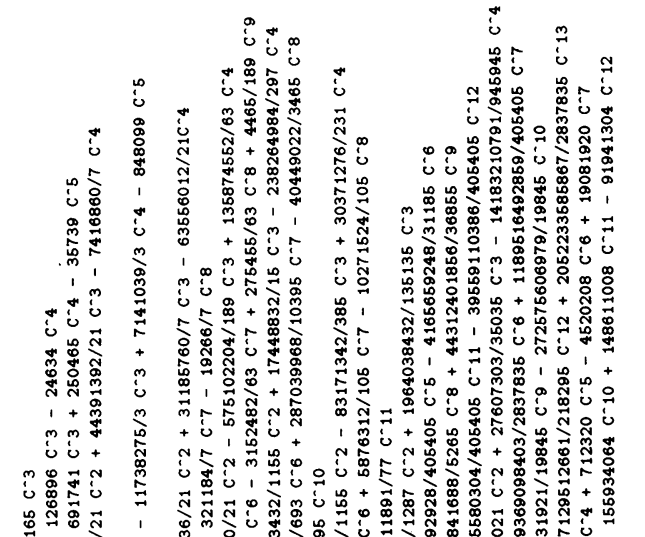

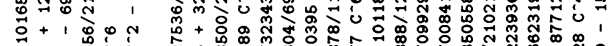

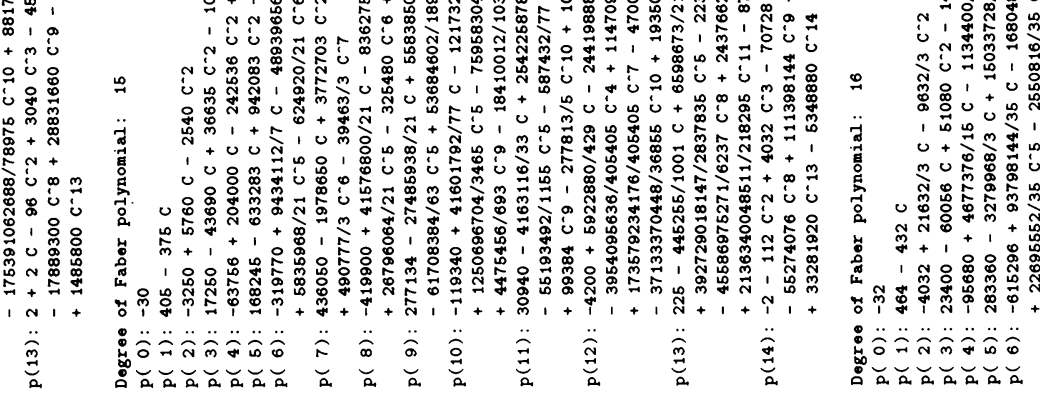

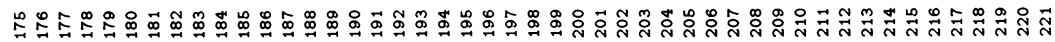
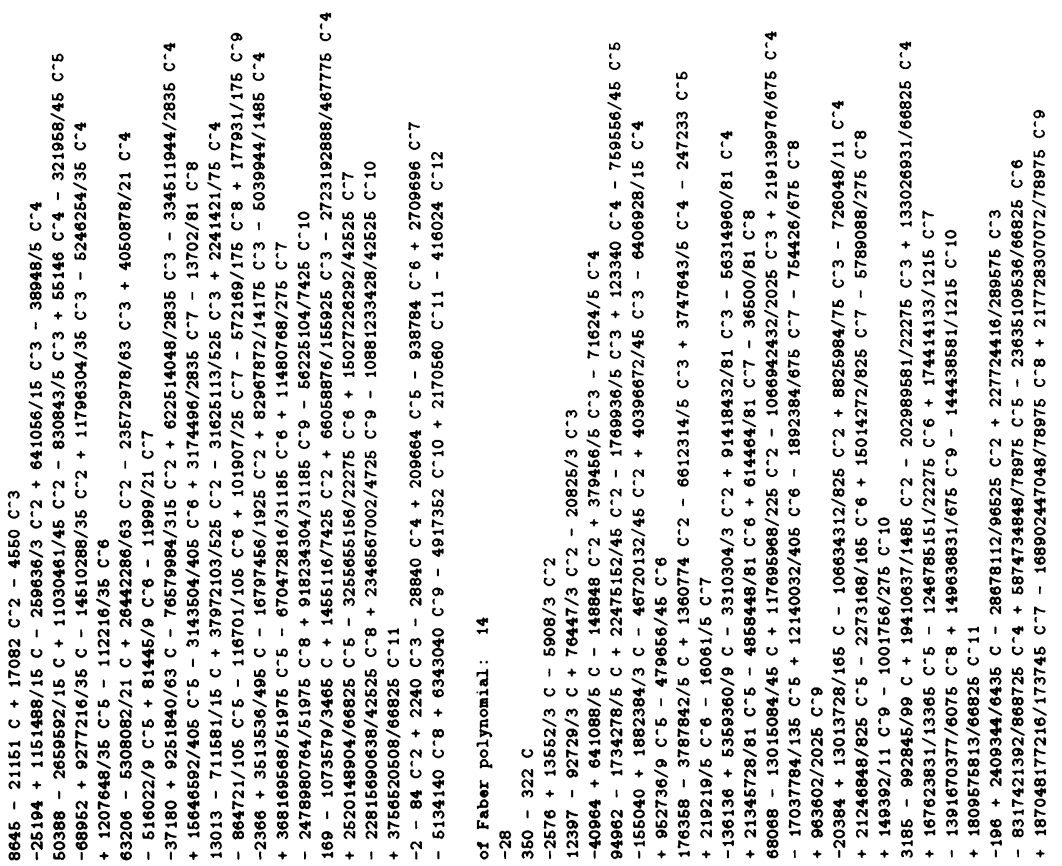

莒芌䒘苍

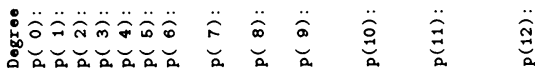

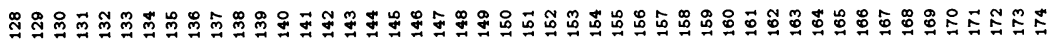



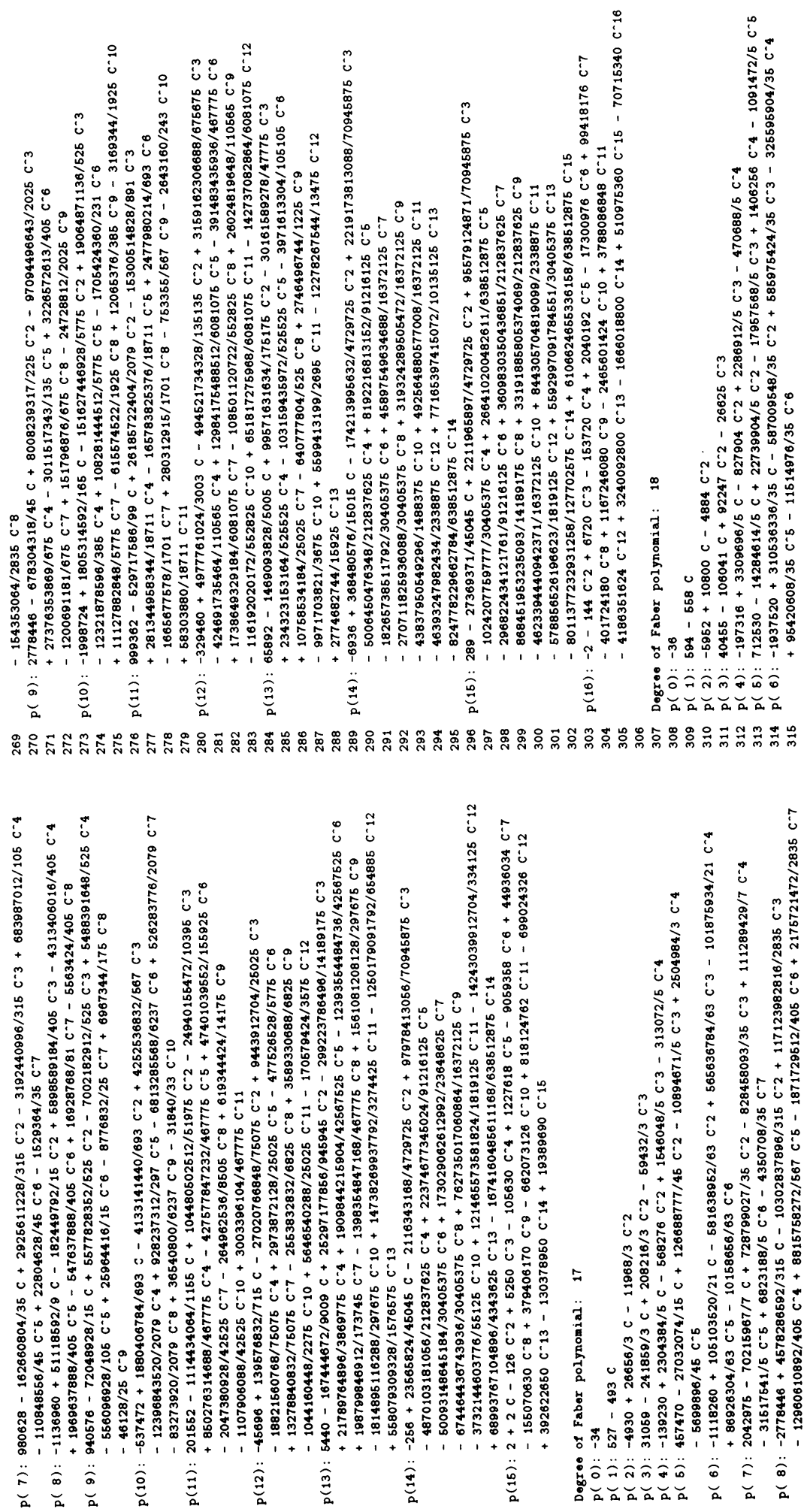

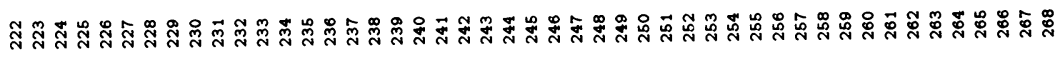




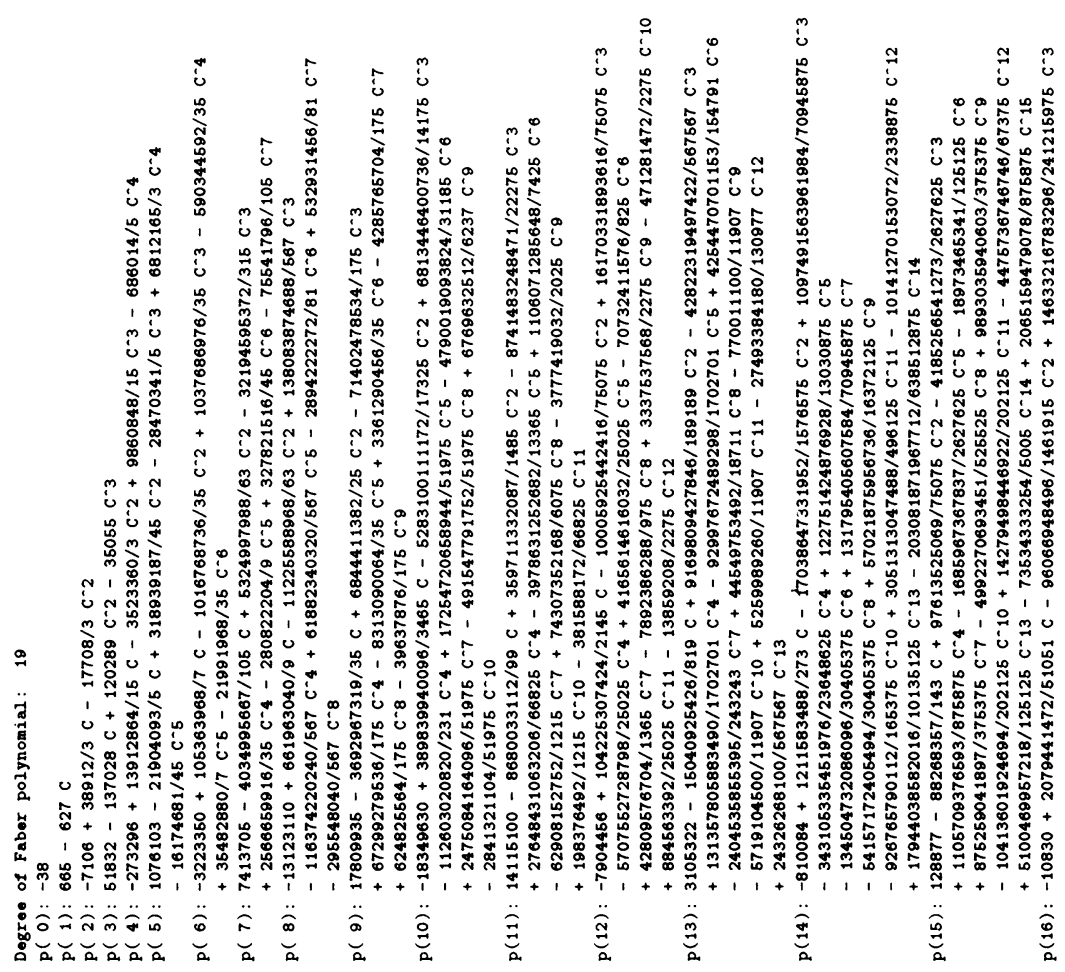

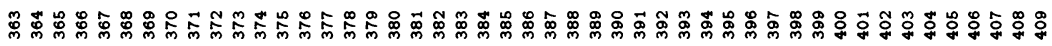

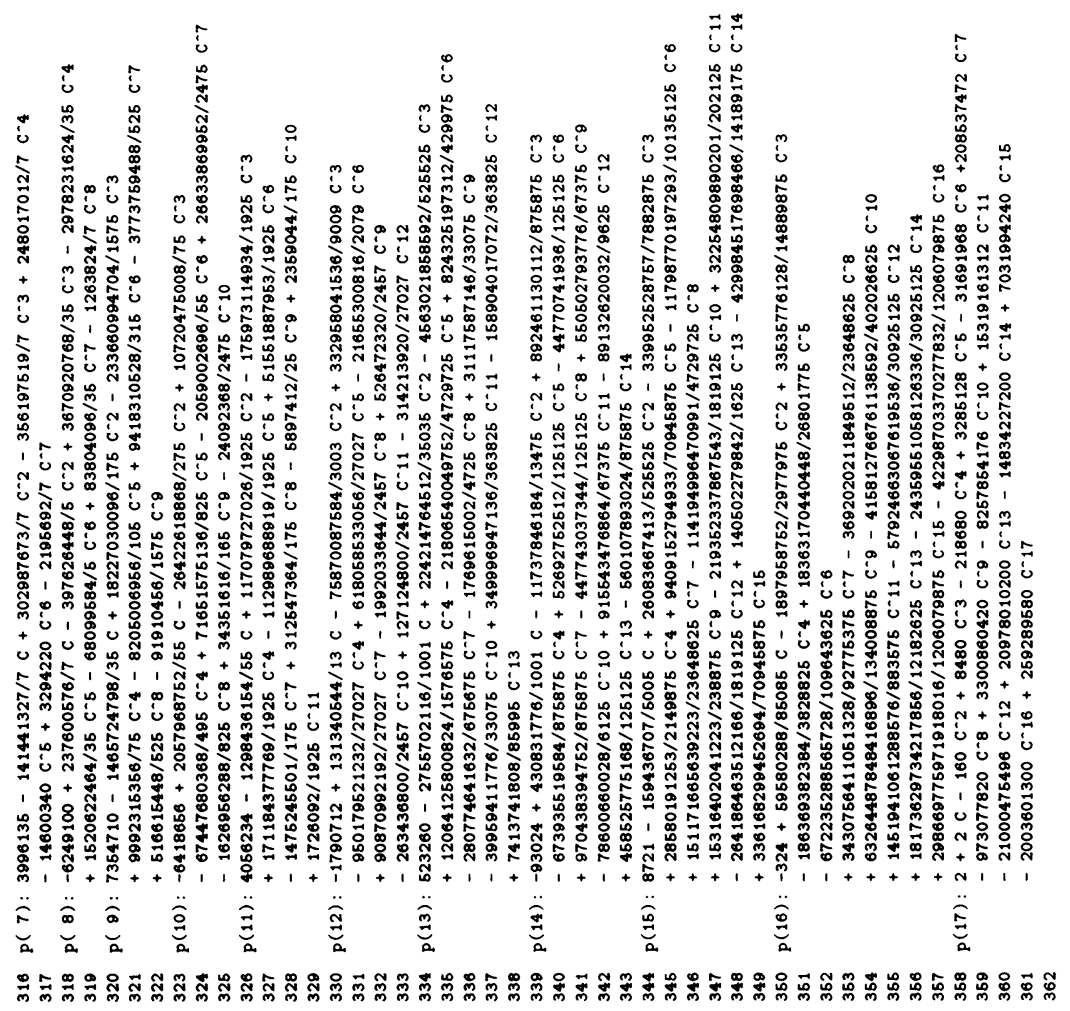



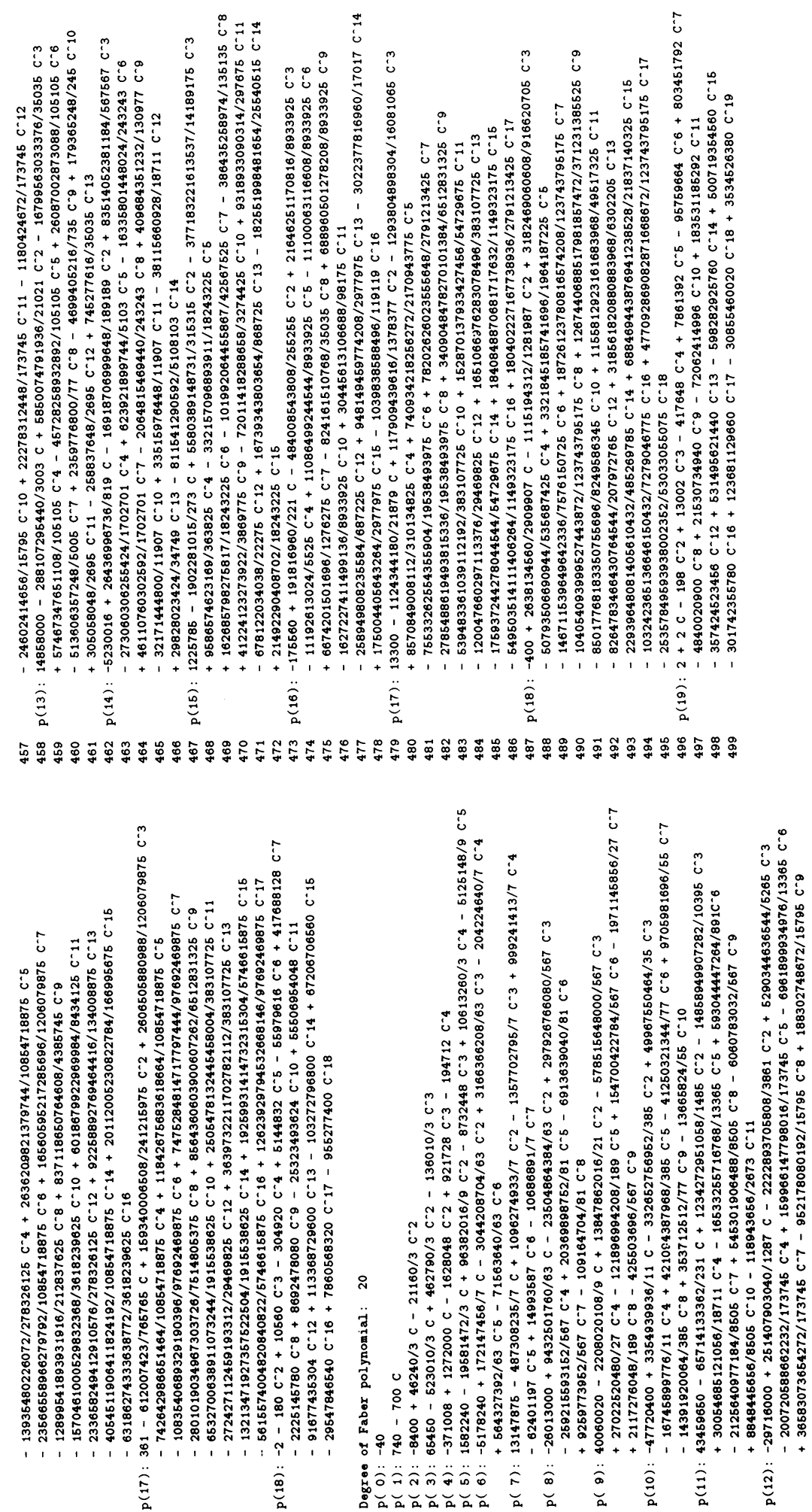